\title{
Ethics of pre-exposure prophylaxis in high-risk HIV patients
}

\author{
Adriana Cappelletti \\ Faculty Reviewer: Chil-Yong Kang, PhD, FRSC (Department of Microbiology and Immunology)
}

\section{ABSTRACT}

Pre-exposure prophylaxis (PrEP) is the daily use of antiretrovirals to prevent human immunodeficiency virus (HIV) transmission in uninfected people at high risk of infection, including men who have sex with men and serodiscordant heterosexual couples. PrEP has the potential benefit of reducing the human population's viral load, and unlike barrier protection, PrEP allows for individual control over HIV exposure. However, ethical concerns have been raised around this intervention's risk of serious side effects. These side effect risks may not be justifiable given PrEP's modest benefit and the existence of standard HIV prevention: condoms, counseling, and education. Critics of this preventative approach also worry that PrEP promotes unsafe sexual practices such as sex without barrier protection. However, this discussion is harmful to the underserviced at-risk groups targeted by PrEP, and prohibition of PrEP for this reason is unjustified in a society that allows women to access oral contraceptives despite the risk of adopting unsafe sexual practices. To ensure the safety of high-risk HIV groups, future clinical trials of PrEP should compare this intervention to the standard of prevention rather than placebo, and stigma mitigation strategies must be implemented at the policy level.

\section{BACKGROUND}

The global disease burden of human immunodeficiency virus (HIV) is tremendous, accounting for approximately 39 million deaths since the onset of the epidemic and currently affecting over 35 million people worldwide. ${ }^{1}$ In Canada, out of approximately $75500 \mathrm{HIV}$ infected people, ${ }^{2}$ more than half of incident HIV infections occur in men who have sex with men (MSM), ${ }^{3}$ and Aboriginal people are disproportionately affected, accounting for $12.5 \%$ of new HIV infections in Canada despite representing only $3.3 \%$ of the population. ${ }^{4}$ Aboriginal women who are injection drug users or sex workers are particularly vulnerable. ${ }^{5}$

For decades, scientists have been unsuccessful in attempts to develop a cure or vaccine for this disease. However, a promising new preventative measure has emerged: repurposing of existing antiretrovirals (ARV) for pre-exposure prophylaxis (PrEP) regimens.

$\operatorname{PrEP}^{*}$ is the daily use of ARV in HIV-uninfected people at high risk of acquiring HIV. ${ }^{6,7}$ Trials of PrEP began in 2005, and the Iniciativa Profilaxis Pre-Exposición (iPrEX) trial of combined oral

*ARV may also be used as "treatment as prevention" (TasP) in HIV-infected people as a means of suppressing viral replication and preventing transmission to their sexual partner. ${ }^{5}$ This article focuses on the ethics surrounding PrEP, a preventative measure in non-infected individuals. tenofovir-emtricitabine (Truvada ${ }^{\circledR}$ ) was the first study to reveal phase III trial results of PrEP's efficacy. The iPrEX trial identified a $44 \%$ decreased risk of HIV acquisition with Truvada ${ }^{\circledR}$ compared to placebo in MSM and transwomen who have sex with men. The pill's efficacy was increased to $73 \%$ with $90 \%$ or greater adherence to the daily Truvada ${ }^{\circledR}$ regimen. ${ }^{8}$

Subsequent PrEP studies demonstrated a $67 \%$ to $75 \%$ reduction in HIV transmission among heterosexual serodiscordant African couples and a $39 \%$ risk reduction when using a vaginal gel form of PrEP.,10 In July 2012, the US Food and Drug Administration approved Truvada ${ }^{\circledast}$ for people at high risk of HIV acquisition, and the World Health Organization (WHO) has set guidelines for use of PrEP by MSM and serodiscordant heterosexual couples. ${ }^{6}$

In the midst of the excitement surrounding PrEP, substantial ethical debates have arisen. This paper will explore two important ethical issues: do the benefits of PrEP outweigh its potential risks? Is it ethical to allocate ARV to non-infected individuals when countless people with HIV remain untreated?

\section{COMPARING BENEFITS AND RISKS OF PREP}

From a utilitarian perspective, PrEP may substantially benefit the global community by reducing the number of new HIV infections and thus the viral load of the population, thereby decreasing the future need of ARV and maximizing the number of lives saved.,111

Unlike other harm reduction strategies for preventing HIV, such as barrier protection, PrEP promotes individual control over HIV exposure. Although adherence to PrEP has been less than perfect in trials, the proper use of PrEP, like the contraceptive pill, depends on the individual taking the medication. In contrast, incorrect use of barrier protection resulting in HIV infection may be attributed to a sexual partner's incorrect use. ${ }^{7}$ This is especially important in vulnerable populations who have difficulty negotiating condoms, such as sex workers or young African women whose husbands have more than one sexual partner., ${ }^{5,711}$ The drawback of this independence, however, is that structural effects of misogyny remain unaddressed. ${ }^{12}$

Despite evidence of PrEP's safety in MSM populations and serodiscordant heterosexual couples, ethicists have raised the question of whether the potential benefits of PrEP outweigh the risks of prescribing drugs with potentially serious side effects to healthy individuals. ${ }^{11,12}$ Truvada $^{\circledR}$ carries the risk of nausea, acute kidney failure, hypophosphatemia, bone mineral density reduction and lactic acidosis. Statistics from the iPrEX trial reveal that for every 100 individuals, Truvada ${ }^{\circledast}$ reduced the number of HIV infections by less than 1, while 10 and 12 developed kidney problems or unintended weight loss, respectively. Are these risks worthwhile when 
condoms, counseling and education exist as the standard of prevention for HIV? Perhaps, if this standard of prevention is failing a population due to reasons such as "collective condom fatigue" or unwillingness to practice safe sex..$^{12}$ At the very least, clinical trials of PrEP should not exclude these behavioural interventions from their placebo arm in order to preserve clinical equipoise, protect study participants from preventable harm, and avoid overestimating the benefits of PrEP. ${ }^{12,13}$

Potentially unfair comparisons of PrEP to placebo are not the only reasons for concern about PrEP's effectiveness; it is also unclear whether the study populations examined reflect the populations in which these drugs will be distributed. In the iPrEX trial, only 227 of 2499 participants were American, despite the fact that the US was the probable catchment area for Truvada ${ }^{\circledR}$. This "offshore trial" led the Centre for Disease Control and Prevention to question whether the results of iPrEX were transferable to the target American population and whether cultural differences among the study sample influenced the results. ${ }^{11}$

\section{DO THE BENEFITS OF PREP OUTWEIGH} ITS POTENTIAL RISKS? IS IT ETHICAL TO ALLOCATE ARV TO NON-INFECTED

\section{INDIVIDUALS WHEN COUNTLESS PEOPLE WITH HIV REMAIN UNTREATED?}

Furthermore, PrEP theoretically carries the risk of increasing HIV drug resistance, though this has fortunately not been found to date. ${ }^{11,13}$

Critics of PrEP have also suggested that it could promote "behavioural disinhibition," the adoption of riskier sexual behaviours such as unprotected sex and having multiple sexual partners due to a belief that ARV completely eliminates the risk of HIV transmission. ${ }^{3,14}$ Interviews of 164 American MSM seronegative couples revealed a significant association between intimacy motivations for condomless sex and the intention to adopt PrEP. ${ }^{15}$ Fortunately, evidence of behavioural disinhibition has yet to be demonstrated in actual PrEP trials. In fact, the Canadian-European IPERGAY PrEP trial of 400 MSM revealed a decreasing median number of sexual partners during the trial period. ${ }^{14}$ Although this statistic was non-significant, it supports that, at the very least, PrEP does not increase risky behaviour. ${ }^{16}$

Unfortunately, it would take many years to acquire strong evidence of clinical efficacy and safety in various specific populations and to rule out the risk of increased resistance or behavioural disinhibition. One could argue that the potential risks of PrEP and potential overestimates of its effectiveness are insufficient reasons for halting a movement towards a preventative approach that could save lives and reduce one of the world's greatest disease burdens.

Moreover, with regards to behavioural disinhibition, one could argue that raising this concern constitutes "inappropriate moralizing about sex" that is discriminatory towards MSM, sex workers and other underserviced high-risk populations. A harmful and heterosexist "PrEP whore" discourse has emerged in the media, referring to MSM who utilize PrEP as sexually promiscuous and irresponsible. ${ }^{14,17,18}$ If the possibility of unsafe sexual behaviour is not considered sufficient for prohibiting access to oral contraceptives for women, one could argue that this risk does not justify prohibition of PrEP. ${ }^{19}$

\section{ETHICAL RESOURCE ALLOCATION OF ARV}

Another ethical concern with PrEP is the appropriateness of allocating ARV to prevention when many HIV-positive individuals lack access to this life-sustaining treatment. The iPrEX trial, for example, distributed 1.3 million Truvada ${ }^{\circledR}$ tablets, which is the equivalent of treatment to failure in approximately $1100 \mathrm{HIV}$-infected individuals. ${ }^{12}$ This allocation of resources may be morally justified on the grounds that HIV is so pervasive in certain countries that treating HIV as it is acquired will never put a stop to this epidemic. ${ }^{7}$ One could argue that society cannot afford to postpone prevention programs that can begin reducing the spread of HIV today.

It is possible that PrEP will actually improve ARV access to infected individuals by propelling improvement of the existing ARV infrastructure, as mother-to-child prevention programs have done.? Furthermore, successful implementation of PrEP programs could save the health system significant costs over time and demonstrate an excellent return on investment. ${ }^{11}$ Despite an estimated cost of $\$ 12$ 000USD per person per year for PrEP, ${ }^{14}$ mathematical modeling suggests that this cost is offset or even negated by future saving in lifelong treatment, especially among high risk groups. ${ }^{20}$

\section{FUTURE DIRECTIONS}

The most cost effective and ideal PrEP would be an effective prophylactic HIV vaccine. Although this is not yet available, the current approach using PrEP has great potential to change the face of HIV. As research and regulations of PrEP advance, steps must be taken to ensure its ethical implementation.

In research, excluding the standard of prevention from clinical trials is only ethically justifiable where trials have incorporated "recalcitrance to condom use" in their inclusion criteria for high-risk study participants. ${ }^{12}$

In terms of policy, culturally tailored stigma mitigation strategies must be developed in order to reduce stigmatizing discourse around PrEP. ${ }^{14}$ For example, survey data of US citizens suggests that public favour of PrEP may be improved by highlighting this intervention's potential overall population benefits, as opposed to focusing public messaging on its benefits to the gay community specifically. ${ }^{17}$ Increased public support for PrEP would promote equitable access for marginalized groups and hopefully mitigate negative social stereotypes associated with ARV. Improving cultural attitudes is essential to creating a safe environment for young queer people trying to educate themselves about safe sex practices in a heteronormative world. ${ }^{18}$

Lastly, the medical community must be prepared to provide culturally safe and trauma-informed care to members of underserviced populations who may benefit from PrEP, such as female 
Aboriginal sex workers, in whom a history of trauma and discrimination is likely. ${ }^{4,5}$ All conversations about PrEP should be complemented by education on safe sex practices and HIV testing, such that the value of condoms in preventing HIV transmission is not underscored..$^{14}$

\section{REFERENCES}

1. World Health Organization [Internet]. Global health observatory (GHO) data: HIV/AIDS; c2016 [cited 2016 Apr 04]. Available from: http://www.who.int/gho/hiv/en/

2. CATIE [Internet]. The epidemiology of HIV in Canada; 2015 [cited 2016 Apr 04]. Available from: http://www.catie.ca/fact-sheets/epidemiology/epidemiology-hiv-canada

3. O'Byrne P, MacPherson P. HIV treatment as prevention in men who have sex with men: examining the evidence. CMAJ. Canada Inc; 2016;188(3):198-203.

4. McCall J, Lauridsen-Hoegh P. Trauma and Cultural Safety: Providing Quality Care to HIV-Infected Women of Aboriginal Descent. J Assoc Nurses AIDS Care. Elsevier Ltd; 2014;25(1):S70-8.

5. Shannon K, Kerr T, Allinott S et al. Social and structural violence and power relations in mitigating HIV risk of drug-using women in survival sex work. Soc Sci Med. 2008;66:911-21.

6. World Health Organization. Guidance on oral pre-exposure prophylaxis (PrEP) for serodiscordant couples, men and transgender women who have sex with men at high risk of HIV: Recommendations for use in the context of demonstration projects. 2012:1-19

7. Haire B, Kaldor JM. Ethics of ARV based prevention: treatment-as-prevention and PrEP. Dev World Bioeth. 2013;13(2):63-9.

8. Grant RM, Lama JR, Anderson PL et al. Preexposure chemoprophylaxis for HIV prevention in men who have sex with men. N Engl J Med. 2010;363(27):2587-99.

9. Baeten JM, Donnell D, Ndase P et al. Antiretroviral prophylaxis for HIV prevention in heterosexual men and women. N Engl J Med. 2012 Aug 2;367(5):399-410.

10. Abdool Karim Q, Abdool Karim SS, Frohlich JA et al. Effectiveness and safety of tenofovir gel, an antiretroviral microbicide, for the prevention of HIV infection in women. Science. 2010;329:1168-74.

11. Gostin LO, Kim SC. Ethical allocation of preexposure. JAMA. 2016;305(2):191-2.

12. Patton C, Kim HJ. The cost of science knowledge and ethics in the HIV pre-exposure prophylaxis trials. J Bioeth Inq. 2012; 9:295-310.

13. Haire B. It's time: the case for PrEP as an active comparator in HIV biomedical prevention trials. J Bioeth Inq. 2015;239-49.

14. Sugarman J, Mayer KH. Ethics and pre-exposure prophylaxis for HIV infection. J Acquir Immune Defic Syndr. 2013;63:135-9.

15. Gamarel KE, Golub SA. Intimacy motivations and pre-exposure prophylaxis (PrEP) adoption intentions among HIV-negative men who have sex with men (MSM) in romantic relationships. Soc Behav Med. $2015 ; 177-86$

16. Sagaon-teyssier L, Suzan-monti M, Demoulin B et al. Uptake of PrEP and condom and sexual risk behavior among MSM during the ANRS IPERGAY trial. AIDS Care. 2016 Mar; 28 Suppl 1; 48-55.

17. Calabrese SK, Underhill K, Earnshaw VA et al. Framing HIV pre-exposure prophylaxis (PrEP) for the general public: how inclusive messaging may prevent prejudice from diminishing public support. AIDS Behav. 2016 Feb 19. [Epub ahead of print]

18. Spieldenner A. PrEP whores and HIV prevention: the queer communication of HIV pre-exposure prophylaxis (PrEP). J Homosex. 2016 Mar
1. [Epub ahead of print]

19. Venter F, Allais L, Richter M. Exposure ethics: does HIV pre-exposure prophylaxis raise ethical problems for the health care provider and policy maker? Bioethics. 2014;28(6):269-74.

20. Hallett TB, Baeten J, Heffron R et al. Optimal uses of antiretrovirals for prevention in HIV-1 serodiscordant heterosexual couples in South Africa: a modelling study. PLoS Med, 8(11):e1001123. 